\title{
A Patient-Specific Methodology for Prediction of Paroxysmal Atrial Fibrillation Onset
}

\author{
Elisabetta De Giovanni ${ }^{1}$, Amir Aminifar ${ }^{1}$, Adrian Luca $^{2}$, Sasan Yazdani ${ }^{2}$, \\ Jean-Marc Vesin ${ }^{2}$, David Atienza ${ }^{1}$ \\ ${ }^{1}$ Embedded System Laboratory (ESL) \\ ${ }^{2}$ Applied Signal Processing Group (ASPG) \\ Swiss Federal Institute of Technology Lausanne (EPFL), Lausanne, Switzerland
}

\begin{abstract}
In spite of the progress in management of Atrial Fibrillation (AF), this arrhythmia is one of the major causes of stroke and heart failure. The progression of this pathology from a silent paroxysmal form (PAF) into a sustained $\mathrm{AF}$ can be prevented by predicting the onset of PAF episodes. Moreover, since AF is caused by heterogeneous mechanisms in different patients, as we demonstrate in this paper, a patient-specific approach offers a promising solution. In this work, we consider two ECG recordings, one close to PAF onset and one far away from any PAF episode. For each patient, we extract two 5-minute ECG segments approximately 20 minutes apart. Next, we train a linear Support Vector Machine (SVM) classifier using patientspecific sets of time- and amplitude-domain features. In particular, we consider the P-waves and the QRS complexes in short windows of 5 consecutive heart beats. Finally, we validate the method on the PAF Prediction Challenge (2001) PhysioNet database predicting the onset with an F1 score of $97.1 \%$, sensitivity of $96.2 \%$ and specificity of $98.1 \%$.
\end{abstract}

\section{Introduction and State-of-the-Art}

Atrial Fibrillation (AF), defined as a quivering or irregular heartbeat, is one of the major causes of stroke and heart failure [1]. The majority of patients suffer from an initial paroxysmal form (PAF), which progresses into a persistent or permanent arrhythmia. Moreover, a significant proportion of patients affected by PAF are initially asymptomatic, even though risk of complications for these patients is similar to the symptomatic condition [1].

According to the European Society of Cardiology (ESC) 2016 guidelines [1] for the management of AF, the aim is to reduce the frequency of episodes, prevent complications and alleviate symptoms, in the case of PAF. Furthermore, PAF patients may be suitable for domiciliary selftreatment, using the so-called "pill in the pocket" approach
[1], that is, a single oral dose pharmacological cardioversion. Therefore, predicting a recent-onset episode of PAF may shorten the initiation of the treatment, as well as the time to resolution of symptoms. Additionally, this prediction is particularly relevant for treating asymptomatic episodes, which remain unseen otherwise.

Different studies describe the prediction of PAF onset, by analysing changes in the surface electrocardiogram (ECG). The classical approach is to consider premature atrial complexes (PACs) and P-wave variability $[2,3]$. PACs are premature beats originating in the atria from ectopic pacemaking tissue active before the sinoatrial node. Zong et al. [2] detect the PACs and predict the PAF onset based on a measurement of PAC rate weighted for different windows in the signal, favouring the closest to the onset. Schreier et al. [3] analyse the P-wave morphology of both regular and premature beats. Then, they extract the probability that a specific degree of P-wave variability is associated with a PAF episode. Other approaches consider the $\mathrm{P}$-wave non-linear dynamics to achieve higher accuracy in the prediction [4]. However, AF is caused by heterogeneous mechanisms in different patients and the therapeutic strategies should derive from the individual conditions. Different works report patient-specific modelling, in particular for ECG signal analysis. Indeed, two works report automatic patient-specific classification of normal or premature beats considering swarm optimization feature selection $[5,6]$.

This paper aims to predict PAF onset while removing inter-patient variability to achieve higher accuracy and potentially optimize individual patient therapy. Section 2 describes in detail the methodology used to tackle this problem. Section 3 presents the results of the methodology applied. Finally, in Section 4 we conclude that it is possible to accurately predict the PAF episodes using our patientspecific methodology. 


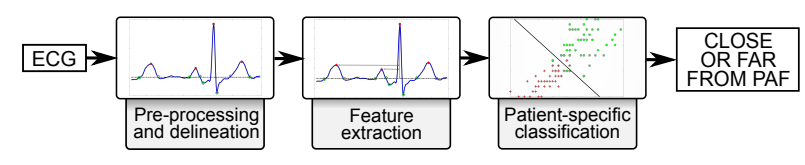

Figure 1. Block diagram of the proposed method

\section{Patient-Specific PAF Prediction}

Figure 1 shows the block diagram describing the steps of our methodology. First, the ECG signal is filtered and the fiducial points are extracted. Secondly, time- and amplitude-domain features related to the P-wave and QRS complex are extracted. Finally, the classification method predicts whether the considered signal is close or far from the PAF onset on each patient.

\subsection{ECG Pre-Processing and Delineation}

The pre-processing of the ECG signal consists in applying a morphological filtering [7], which removes the baseline wandering. Then, we extract the fiducial points using Pan-Tompkins QRS complex detection [8]. Next, we detect the peak of the P-wave using the average durations of the QRS complexes, $\mathrm{P}$-waves and T-waves. The onset and offset of the P-wave are defined as the initial increase from the isoelectric line and the return to it. First, we consider the Euclidean distance between the P-wave and triangle waves constructed with the P-wave peak and consecutive points on the isoelectric line. Then, we choose the onset (or offset) as the point approximately on the isoelectric line where the Euclidean distance is minimum. Figure 2 shows the triangle wave that gives the minimum distance from the P-wave, and the onset and offset detected.

\subsection{Feature Extraction}

The fiducial points used for feature extraction are the onset, peak and offset of the P-wave, R-wave, and S-wave. We choose time- and amplitude domain features in short windows of 5 consecutive beats. The time-domain features correspond to time intervals from each fiducial point to the $\mathrm{R}$ peak in the same beat, within the short window. Moreover, we consider the RR interval between two beats. The amplitude-domain features correspond to the voltage value for each fiducial point relative to the isoelectric line.

\subsection{Patient-Specific Classification}

The patient-specific learning approach consists in training a classifier on two ECG signals close and far from PAF onset for each patient and testing on new examples from the same patient. Assuming that the conditions in a signal close to (or far from) the onset are the same, we extract the training and test set. We choose two segments at least 20

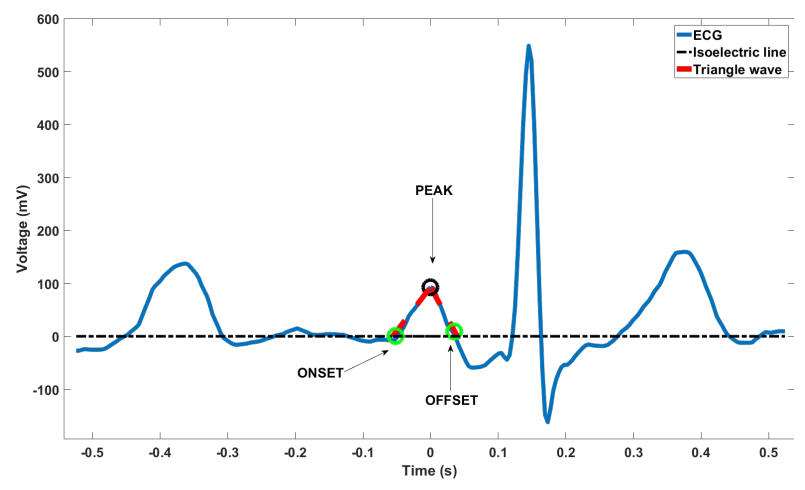

Figure 2. Detection of the onset (offset) of the P-wave based on the minimum euclidean distance between the Pwave and a triangle wave with a slope depending on the peak and the onset (offset).

minutes apart, considering the last $350 \mathrm{R}$ peaks (around 5 minutes) as the training set and the first $350 \mathrm{R}$ peaks (discarding the beginning of the signal, in case of initial noise) as the test set. The window length is chosen based on a trade-off between number of samples to avoid overfitting and classification accuracy.

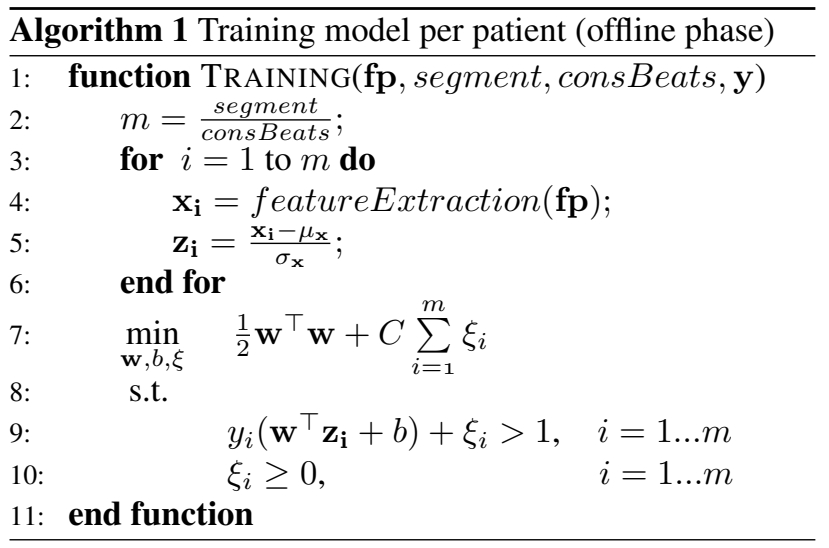

As shown in Algorithm 1, the training part takes as input (Line 1) the fiducial points fp extracted with the delineation, the segment (in this case 350 beats) extracted at the end of the two ECG signals close and far from PAF, the short window of consecutive beats (consBeats, in this case 5), and the labels $\mathbf{y}$. Then, Line 2 computes the number of samples in the training set, $m$. Next, in Lines 3-6 the algorithm extracts the features reported in Section 2.2 within the segment and normalizes them. Next, Lines 7-10 compute the training model considering an SVM classifier with a linear kernel. The SVM linear model is the separating hyperplane between the two classes and is described as a linear combination of the normalized features vector $\mathbf{z}$ and the normal vector to the hyperplane $\mathbf{w}$. Since the classes are not linearly separable, a soft-margin technique 
is applied considering correction parameters $(C$ and $\xi)$ to compensate for the examples on the wrong side of the margin.

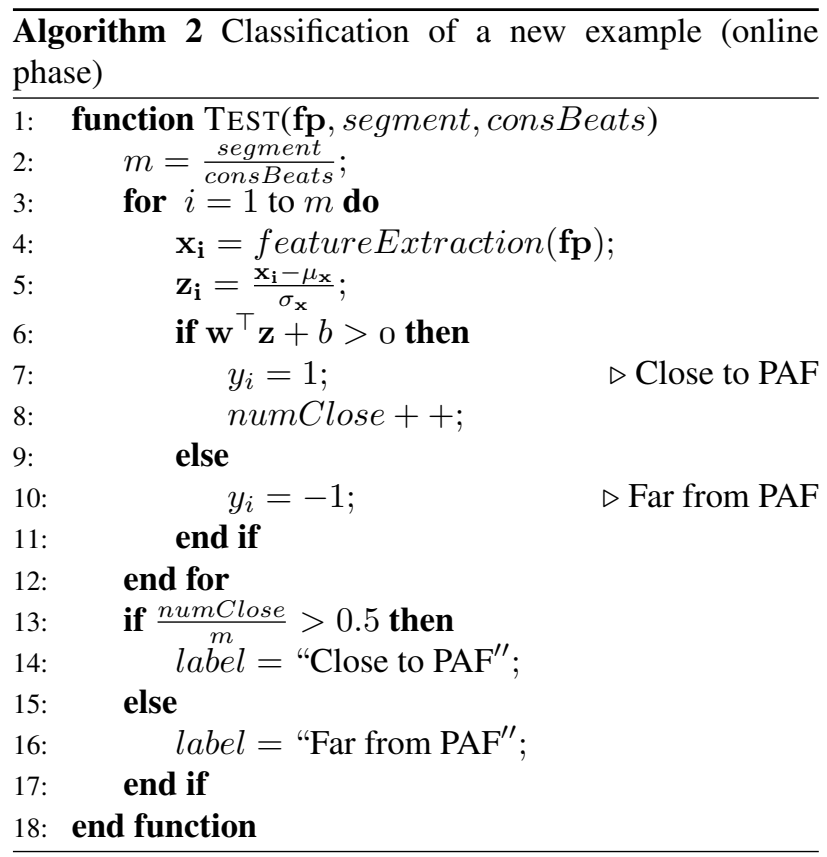

Algorithm 2 considers a new segment of 350 beats and applies the same features extraction and normalization as the training (Lines 1-5). Then, given the training model, the algorithm classifies a short window as 1 or -1 (Lines 6-11). Meanwhile, it counts the number of windows classified as 1. As shown in Lines 13-17, if the number of windows represents more than $50 \%$ the total amount of samples, the whole 5-minute window is classified as "Close to PAF", otherwise "Far from PAF".

\section{Experimental Setup and Results}

We validate our approach on the PAF Prediction Challenge (2001) PhysioNet database $[9,10]$. The dataset includes 53 patients affected by PAF. For each patient two 30-minute ECG signals were recorded at a sampling frequency of $128 \mathrm{~Hz}$ : one right before a PAF onset and one at least 45 minutes far from any PAF episodes. For reasons related to the morphological filtering and delineation, we resample the signals at $250 \mathrm{~Hz}$ and we choose automatically one of the two channels available in the dataset (related to lead II). The data for each patient are divided considering the method described in Section 2. The training data includes 350 beats extracted from the end of each signal. We test the approach on segments of 350 beats at the beginning of the signals, 20 minutes apart from the training data. This setup allows to predict 25 minutes before the PAF.

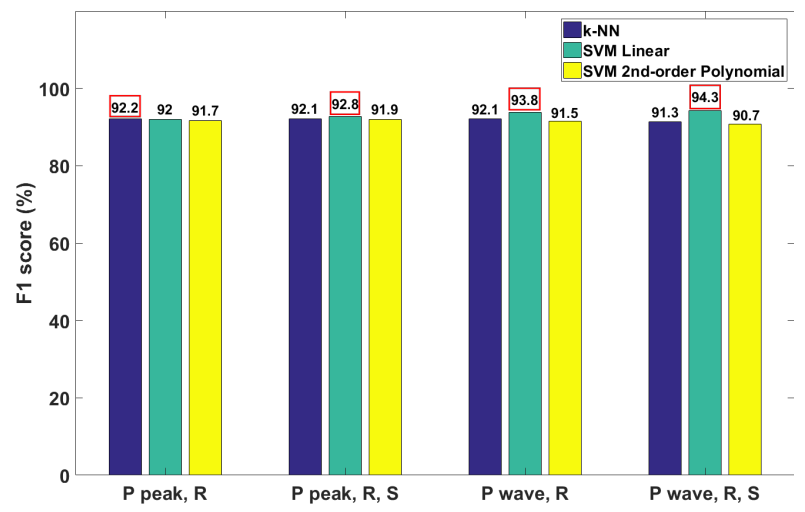

Figure 3. Results of leave-one-out cross-validation for three different classifiers applied to four sets of features for the patient-specific classification. F1 score is computed as a measure of overall accuracy.

First, we perform a leave-one-out Cross-Validation (CV) on the training set for each patient to evaluate the generalization performance of different classifiers and select the combination of features that best predicts the output. Then, we test the approach considering the results obtained using CV. We examine different combinations of QRS complex and P-wave features, as described in Section 2.2. Moreover, we analyse k-nearest neighbours (k-NN) and support vector machine (SVM) classifiers, the last one with different kernels (first and second order polynomial). Finally, we evaluate the diagnostic ability of the classifiers on the test set with the F1 score, which focuses on the positive rate, sensitivity and specificity.

\subsection{Leave-One-Out Cross-Validation}

As reported in Figure 3, we apply first the leave-oneout $\mathrm{CV}$ on different classifiers and compute the $\mathrm{F} 1$ score for all patients. The CV results show that the classifiers have similar values for the same set of features, while the results improve considering different sets of features for each patient.

\subsection{Patient-Specific Classifier Stability}

In order to analyse the robustness of our results, we perform a 5-fold CV and compare it to the leave-one-out. This comparison allows to analyse the classifier stability based on the variation of number of samples. $\mathrm{k}-\mathrm{NN}$ is sensitive to the local shape of the data and to the training set size and shows a variation of F1 score of $3 \%$. Alternatively, SVM is more robust against local minima with a variation of only $0.15 \%$ and $0.48 \%$, respectively for linear and quadratic. However, our patient-specific method shows higher accuracy even considering different classifiers and, in particular, the low complex linear SVM classifier. 


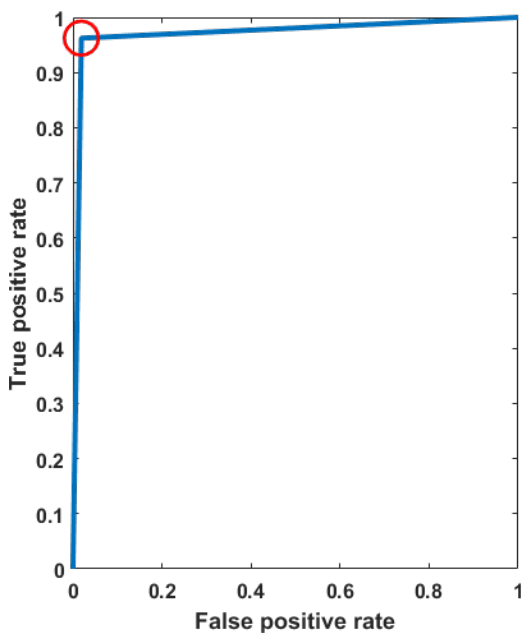

Figure 4. Results of prediction on new examples shown in a ROC curve using the model derived from a linear SVM classifier and the patient-specific feature set selection.

\subsection{Patient-Specific Feature Set Selection}

Our proposed patient-specific approach refers to selecting combinations of features for each patient. These combinations include different fiducial points (cf. Section 2.2). Based on the leave-one-out $\mathrm{CV}$, we choose for each patient the feature set that results in the maximum F1 score. As an example, for one patient, choosing the feature set including the peak of P-wave and R leads to an F1 score of $61.1 \%$, whereas adding also the onset, offset and $\mathrm{S}$ leads to an F1 score of $95.0 \%$. For another patient, the results for choosing the same feature set report an F1 score of $82.3 \%$, but adding onset and offset reaches an F1 score of $88.9 \%$.

\subsection{Evaluation on Test Set}

Considering the linear SVM classifier and by selecting the feature set for each patient, we test our approach on new examples included in the test set. We evaluate the classification considering the F1 score, sensitivity and specificity. Figure 4 shows the ROC curve of the linear SVM for the patient-specific selection of the best feature set. Finally, considering all the patients in the dataset, our method is able to predict PAF onset with an F1 score of $97.1 \%$, sensitivity of $96.2 \%$, and specificity of $98.1 \%$, which is higher than other approaches that only consider inter-patient variability (79\% [2] and 93\% [4]).

\section{Conclusion}

Patients affected by PAF are at risk of the arrhythmia progression into a sustained AF. Therefore, predicting the onset of PAF episodes is required for progression prevention. Moreover, PAF prediction can lower stroke risk. In this paper, we demonstrate that considering the specific profile of each patient, highly improves PAF onset prediction. By training a linear SVM classifier and considering features related to the P-wave and the QRS complex, our patient-specific method predicts PAF onset with an F1 score of $97.1 \%$, sensitivity of $96.2 \%$ and specificity of $98.1 \%$.

\section{Acknowledgements}

This work has been partially supported by the Hasler Foundation (project no. 15048), and RTD project ObeSense (no. 20NA21 143081) evaluated by the Swiss NSF and funded by Nano-Tera.ch with Swiss Confederation financing.

\section{References}

[1] Kirchhof P, et. al. 2016 ESC Guidelines for the management of atrial fibrillation developed in collaboration with EACTS. European Heart Journal 2016;37:2893-2962.

[2] Zong W, et al. A methodology for predicting paroxysmal atrial fibrillation based on ECG arrhythmia feature analysis. In Computers in Cardiology 2001. Vol.28 (Cat. No.01CH37287). IEEE, 2001; 125-128.

[3] Schreier G, et al. An automatic ECG processing algorithm to identify patients prone to paroxysmal atrial fibrillation. In Computers in Cardiology 2001. Vol.28 (Cat. No.01CH37287). IEEE, 2001; 133-135.

[4] Martínez A, et al. Alteration of the P-wave non-linear dynamics near the onset of paroxysmal atrial fibrillation. Medical Engineering Physics 2015;37:692-697.

[5] Das MK, et al. Patient-specific ECG beat classification technique. Healthcare technology letters 2014;1:98-103.

[6] Ince $\mathrm{T}$, et al. Automated patient-specific classification of premature ventricular contractions. In 2008 30th Annual International Conference of the IEEE Engineering in Medicine and Biology Society. IEEE, 2008; 5474-5477.

[7] Sun Y, et al. ECG signal conditioning by morphological filtering. Computers in biology and medicine 2002;32:46579.

[8] Pan J, et al. A Real-Time QRS Detection Algorithm. IEEE Transactions on Biomedical Engineering 1985;32:230-236.

[9] Goldberger AL, et al. PhysioBank, PhysioToolkit, and PhysioNet: components of a new research resource for complex physiologic signals. Circulation 2000;101:E215-20.

[10] Moody G, et al. Predicting the onset of paroxysmal atrial fibrillation: the Computers in Cardiology Challenge 2001. In Computers in Cardiology 2001. Vol.28 (Cat. No.01CH37287). IEEE, 2001; 113-116.

Address for correspondence:

Elisabetta De Giovanni

EPFL STI IEL ESL - ELG 133 - Station 11

CH-1015 Lausanne, Switzerland

E-mail address: elisabetta.degiovanni@epfl.ch 